\title{
Visual Fixation Assessment in Patients with Disorders of Consciousness Based on Brain-Computer Interface
}

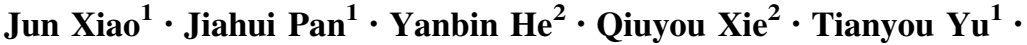 \\ Haiyun Huang ${ }^{1}$ Wei $\mathrm{Lv}^{2} \cdot$ Jiechun $\mathrm{Zhang}^{2} \cdot$ Ronghao $\mathrm{Yu}^{2} \cdot$ Yuanqing $\mathrm{Li}^{1}$
}

Received: 28 January 2018/ Accepted: 29 May 2018/Published online: 17 July 2018

(C) The Author(s) 2018

\begin{abstract}
Visual fixation is an item in the visual function subscale of the Coma Recovery Scale-Revised (CRS-R). Sometimes clinicians using the behavioral scales find it difficult to detect because of the motor impairment in patients with disorders of consciousness (DOCs). Braincomputer interface (BCI) can be used to improve clinical assessment because it directly detects the brain response to an external stimulus in the absence of behavioral expression. In this study, we designed a BCI system to assist the visual fixation assessment of DOC patients. The results from 15 patients indicated that three showed visual fixation in both CRS-R and BCI assessments and one did not show such behavior in the CRS-R assessment but achieved significant online accuracy in the BCI assessment. The results revealed that electroencephalography-based BCI can detect the brain response for visual fixation. Therefore, the proposed $\mathrm{BCI}$ may provide a promising method for assisting behavioral assessment using the CRS-R.
\end{abstract}

Keywords Visual fixation - Brain-computer interface . Disorder of consciousness · Coma recovery scale-revised . Electroencephalography

Jun Xiao and Jiahui Pan have contributed equally to the manuscript.

Ronghao Yu

gesund@21cn.com

$\triangle$ Yuanqing Li

auyqli@scut.edu.cn

1 Center for Brain Computer Interfaces and Brain Information Processing, South China University of Technology, Guangzhou 510640, China

2 Coma Research Group, Centre for Hyperbaric Oxygen and Neurorehabilitation, General Hospital of Guangzhou Military Command, Guangzhou 510010, China

\section{Introduction}

Patients with severe brain injury may suffer from disorders of consciousness (DOCs), including coma, vegetative state (VS), and minimally conscious state (MCS). Accurate assessment of the status of DOC patients is important for monitoring the effects of treatment and the rehabilitation process. The proposed behavioral scales for DOC patients include the Coma Recovery Scale-revised (CRS-R), the Glasgow Coma Scale, the Full Outline of Unresponsiveness, the Wessex Head Injury Matrix, and the Sensory Modalities Assessment and Rehabilitation Technique. Among them, the CRS-R provides a more fine-grained assessment and has demonstrated significant sensitivity in differentiating MCS from VS patients [1]. The CRS-R contains 6 subscales addressing auditory, visual, motor, oromotor, communication, and arousal processes [1]. Each subscale consists of several items that are critical for identifying subtle behavioral markers of recovery of consciousness, and there are a total of 23 hierarchicallyarranged items. The visual subscale includes five items: visual startle, visual fixation, visual pursuit, object localization, and object recognition, corresponding to scores of $1,2,3,4$, and 5 , respectively [2-4].

Visual fixation plays a key role in the differentiation between VS and MCS, and there are many behavioral scales available for its assessment in patients with DOCs. Sustained visual fixation is considered as present when the eyes change from an initial fixation point and refixate on a new target location for $>2 \mathrm{~s}$ in the CRS-R criteria. However, it is still a matter of debate whether visual fixation indicates automatic subcortical processing or higher-order cortical processing that heralds consciousness. And the different stimuli used in different behavioral scales may affect the accuracy of the 
diagnosis. Therefore, visual fixation assessment has been intensively studied in DOC patients [5-7]. Bruno et al. studied cerebral metabolism in ten DOC patients, five in a VS without fixation and five presenting visual fixation but otherwise all criteria typical of the VS. Patients without fixation showed metabolic dysfunction in a widespread fronto-parietal cortical network and this did not differ from the brain function seen in patients with visual fixation. Recovery rates did not differ between patients without or with fixation. Their findings suggested that sustained visual fixation in DOC patients does not necessarily reflect consciousness and higher-order cortical brain function [5]. Di et al. assessed visual fixation in 81 MCS or VS patients, and the occurrence of fixation to different stimuli was analyzed with the $\chi^{2}$ test. The results showed that $40(49 \%)$ of the 81 patients showed fixation to visual stimuli. Among these, significantly more patients $(39,48 \%)$ showed visual fixation elicited by a mirror compared to a ball $(23,28 \%)$ and a light $(20$, $25 \%$ ). Therefore, they concluded that using a mirror to test visual fixation is a sensitive and accurate method [6]. Naro et al. evaluated the visuomotor integration (VMI) and visual P300 in DOC patients in order to detect residual visuomotor network functionality that potentially sustains aware visual fixation. They found that the MCS patients showed preserved patterns of VMI and P300, whereas nearly all VS patients had no significant VMI. Nonetheless, two fixating VS individuals had a VMI similar to MCS patients. Their data suggest that some VS patients showing visual fixation can be aware [7]. However, the assessment of visual fixation is still a challenging task. First, DOC patients are severely lack of behavioral responses to external stimuli because of motor impairment, such that unambiguous signs of fixation can be difficult to observe or are easily missed [8, 9]. Second, visual responsive behavior for different stimuli has been shown to differ greatly [6]. Moreover, there are problems of subjectivity and misinterpretation of the behavioral response on the part of examiners [10]. Therefore, the challenge of behaviorally assessing visual fixation may result in frequent misdiagnosis in DOC patients as reported in previous studies [11-14].

To overcome the above limitations, brain-computer interfaces (BCIs), which directly detect the endogenous brain responses to external stimuli in the absence of behavioral expression, may provide a solution to improve clinical assessment and complement classical behavioral observations [9]. Our previous studies applied BCIs to assist the assessment of two items in the CRS-R scale: auditory startle and communication $[15,16]$. To further expand that study, here we propose a visual BCI system to detect visual fixation in DOC patients.

\section{Materials and Methods}

\section{Participants}

Five healthy participants (four males; $29 \pm 5$ years old) first participated in the $\mathrm{BCI}$ experiments to validate the proposed BCI system. Next, 15 DOC patients (13 males and 2 females, 13-73 years old) from the General Hospital of Guangzhou Military Command were assessed by BCI and CRS-R (Table 1), and the clinical diagnosis was made according to the CRS-R. The CRS-R evaluation was performed one week before the BCI experiment. Each evaluation consisted of five repeated CRS-R assessments on separate days of a week. For each patient, the CRS-R scores were based on his/her best responses in the repeated CRS-R assessments. The CRS-R scores are not given for patient 15 because he had locked-in syndrome.

The experimental protocols for the DOC patients were approved by the Ethics Committee of the General Hospital of Guangzhou Military Command, and complied with the Code of Ethics of the World Medical Association (Declaration of Helsinki). Written informed consent and authorization to publish individual details in this manuscript were obtained from the healthy participants and the patients' legal surrogates.

\section{Data Acquisition}

A SynAmps ${ }^{2}$ amplifier (Neuroscan Compumedics, El Paso, TX) was used to record scalp EEG signals at a sampling rate of $250 \mathrm{~Hz}$ and the signals were filtered with a bandpass filter between $0.05 \mathrm{~Hz}$ and $100 \mathrm{~Hz}$. A 32-channel EEG cap (LT37) that followed the standard 10-20 system was worn by each participant and the EEG signals were referenced to the right mastoid. The impedances of all electrodes were $<5 \mathrm{k} \Omega$ during data acquisition.

\section{Graphical User Interface and Experimental Paradigms}

Each participant was seated in a comfortable chair (a wheelchair for patients) $0.5 \mathrm{~m}$ from the monitor, which was $27.38 \mathrm{~cm}$ high and $48.7 \mathrm{~cm}$ wide. The graphical user interface (GUI) consisted of a square in the center of the screen (Fig. 1). The side length of the square was $27.38 \mathrm{~cm}$ (corresponding to a visual angle of $28.7^{\circ}$ ), equal to the height of the monitor. In this area, four buttons were arranged at the edge of the square in four locations: left, right, above and below. In the CRS-R-based behavioral assessment, the clinician moved a mirror to one of four locations (right, left, above, and below) in a plus-shaped tracking pattern. Therefore, four buttons rather than some 
Table 1 Summary of clinical status of patients.

\begin{tabular}{lllllll}
\hline $\begin{array}{l}\text { Patient } \\
\text { index }\end{array}$ & Age & Gender & Etiology & $\begin{array}{l}\text { Time since insult } \\
\text { (months) }\end{array}$ & $\begin{array}{l}\text { Clinical } \\
\text { diagnosis }\end{array}$ & $\begin{array}{l}\text { CRS-R score (auditory-visual-motor-oromotor-communica- } \\
\text { tion-arousal) }\end{array}$ \\
\hline Patient 1 & 72 & Male & NTBI & 4 & VS & $4(1-0-1-0-0-2)$ \\
Patient 2 & 16 & Male & TBI & 2 & VS & $5(1-0-2-0-0-2)$ \\
Patient 3 & 52 & Male & NTBI & 6 & VS & $5(1-1-0-1-0-2)$ \\
Patient 4 & 35 & Female & NTBI & 3 & VS & $5(1-0-1-1-0-2)$ \\
Patient 5 & 40 & Male & NTBI & 3 & VS & $6(1-1-1-1-0-2)$ \\
Patient 6 & 33 & Male & TBI & 5 & VS & $7(1-1-2-1-0-2)$ \\
Patient 7 & 32 & Male & NTBI & 3 & VS & $7(1-0-2-2-0-2)$ \\
Patient 8 & 52 & Male & NTBI & 4 & VS & $7(1-1-2-1-0-2)$ \\
Patient 9 & 20 & Male & NTBI & 6 & MCS & $9(1-1-4-1-0-2)$ \\
Patient 10 & 13 & Male & TBI & 2 & MCS & $9(1-1-4-1-0-2)$ \\
Patient 11 & 45 & Male & NTBI & 1 & MCS & $10(1-3-3-1-0-2)$ \\
Patient 12 & 69 & Female & NTBI & 10 & MCS & $11(1-3-5-0-0-2)$ \\
Patient 13 & 61 & Male & TBI & 4 & MCS & $15(2-5-5-1-0-2)$ \\
Patient 14 & 42 & Male & NTBI & 6 & EMCS & $20(4-5-6-1-2-2)$ \\
Patient 15 & 73 & Male & NTBI & 12 & LIS & - \\
\hline
\end{tabular}

LIS, locked-in syndrome; MCS, minimally-conscious state; EMCS, emerging from MCS; NTBI, non-traumatic brain injury; TBI, traumatic brain injury; VS, vegetative state. In the CRS-R score column, the second numbers in brackets are the sub-scores of visual function. A value of 2 in the second sub-score means that the patient showed visual fixation in the behavioral assessment.

other number were designed into the GUI to keep the BCI assessment as close as possible to the CRS-R-based behavioral assessment. One of the four buttons was pseudo-randomly chosen as a target location, and then a button appeared in the center of the square and moved to the target location to guide the patient to re-fixate the new target button. The foreground of all buttons was presented as pictures of a brightly-colored ball, while the background was traditional black. All pictures were cropped to squares according to the full size of the monitor screen, e.g., $4 \times 4$ $\mathrm{cm}^{2}$ in this study.

The paradigm of the proposed BCI system simulated the behavioral assessment of visual fixation in the CRS-R, in which the patient's eyes change from an initial point to a new target location and re-fixate on the new location for $>2$ s. As shown in Fig. 1, each experimental trial began with the visual prompt of a cross lasting $2 \mathrm{~s}$, a target location was randomly chosen from the four directions, and a brightly-colored ball appeared in the middle of the GUI to guide the patient. Then the middle ball moved from the center to the target location, while the four buttons at the edge of the GUI did not move. The movement lasted $2.5 \mathrm{~s}$ and the distance between the center point of the button's initial location and the center point of the new target location was $11.5 \mathrm{~cm}$ (corresponding to a visual angle of $13.1^{\circ}$ ). After the moving ball reached the target location and overlapped with the corresponding button, the four buttons flashed from the foreground to the background in random order, the background appearing for $100 \mathrm{~ms}$ at an inter-stimulus interval of $100 \mathrm{~ms}$. In the stimulation round, each button flashed once. Each experimental trial included ten repetitions of stimulation. When the ten repetitions were finished $(8 \mathrm{~s})$, the BCI algorithm used the EEG data to determine the button on which the subject re-fixated. If the target was detected, which implied that the target was correctly followed and refixated, a tick was presented for $2.5 \mathrm{~s}$ in the center of the GUI. However, no feedback was given for an incorrect result. The time course of a trial is illustrated in Fig. 1. After the trial finished, there was a break of 1-10 s before the next trial began. During the experiment, the patient was verbally encouraged to fixate on the moving target and to avoid decreased arousal as assessed by the clinician.

\section{Experimental Procedures}

First, five healthy individuals participated in the BCI experiment to validate the proposed system. Each participant completed two sessions of the BCI experiment. Each session consisted of two blocks, a calibration block of 10 trials and an online block of 10 trials.

Subsequently, 15 DOC patients participated in two different visual fixation assessments: CRS-R-based behavioral assessment and BCI-based assessment. (1) The CRS$\mathrm{R}$ assessment was conducted by an experienced clinician. 


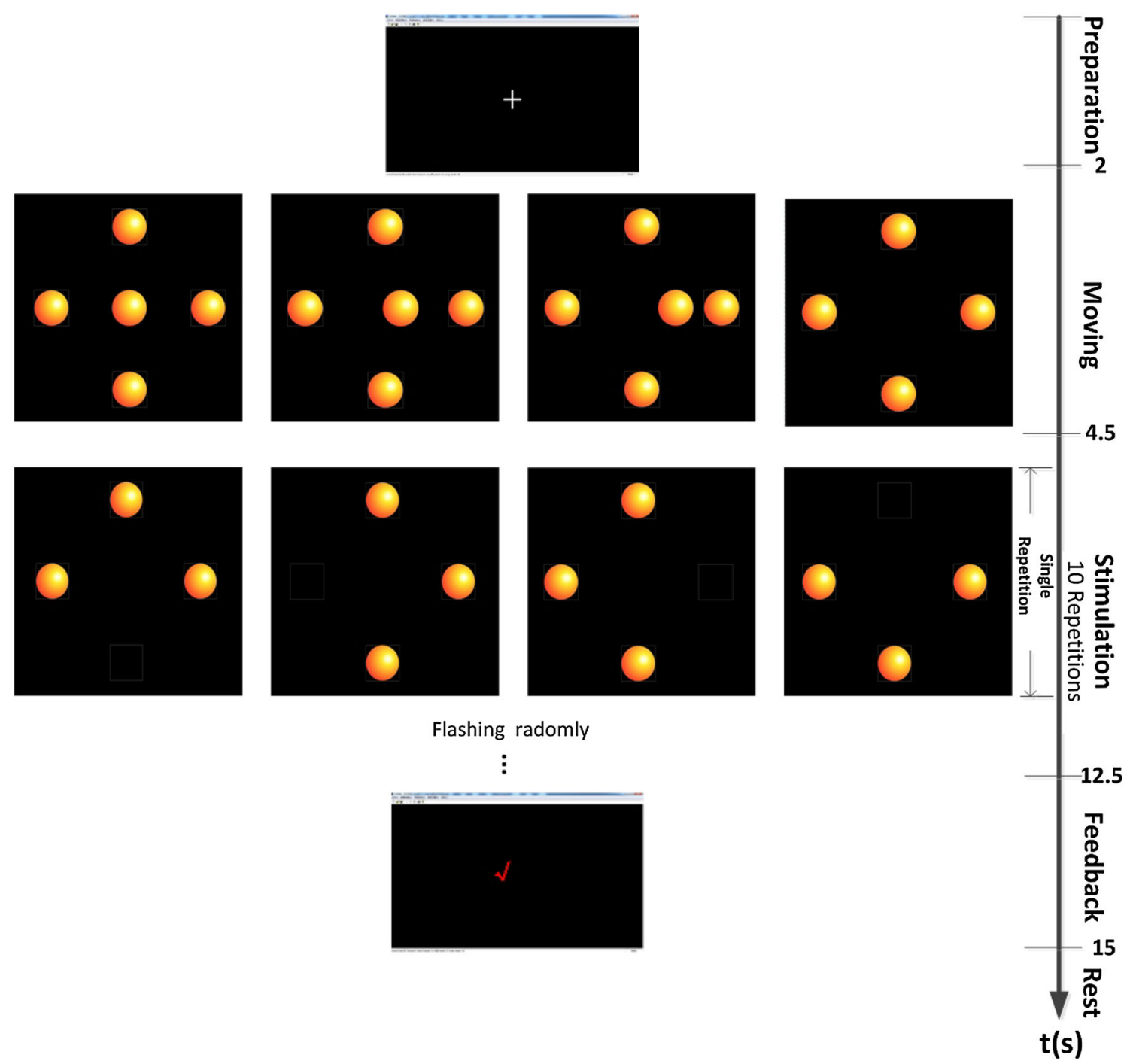

Fig. 1 The GUI and the paradigm of the experiment. A square area was set and four brightly-colored ball buttons were arranged at the edge of the square in four locations. A trial was started with " + " and a ball in the middle of the GUI moved to guide the patient to the new

Following the standard protocol, the clinician held a brightly-colored ball $15-20 \mathrm{~cm}$ in front of the patient's face and then rapidly moved to the upper, lower, right, and left visual fields for a total of 4 trials. The criterion was that the patient's eye changed from the initial fixation point and refixated on the new target location for $>2 \mathrm{~s}$. If at least 2 episodes of fixation were achieved by the patient, he/she was scored 2 for the visual fixation item, otherwise, the lower item of visual startle (item score of 1) was given. (2) The BCI-based assessment was conducted for the 15 patients using the same BCI system as for the healthy participants. Each patient also completed two sessions of the $\mathrm{BCI}$ experiment as described above. target location that was chosen randomly from the four directions. When the ball overlapped with the target button, the four buttons flashed randomly. The stimulation contained 10 repetitions of the flash.

\section{EEG Data Analysis}

Different event-related potential (ERP) components are often used by BCI algorithms to select a target from several interferences, i.e., the other three non-target buttons. According to previous studies [17, 18], ERP features can be constructed from filtered EEG signals, and linear or nonlinear classifiers can be used to determine whether the target is fixated.

The general ERP-based BCI paradigm includes one target and $K-1$ interferences ( $K$ stimuli in total). $X=$ $\left(X^{(1)}, \ldots, X^{(K)}\right)$ denotes the EEG data epoch after stimulus onset. In $X^{(l)} \in R^{C \times T}, \mathrm{C}$ and $\mathrm{T}$ indicate the channels and 
time points respectively. If $a \in(1, \ldots, K)$ is the real target on which the participant fixates, identifying the target from the interferences can be described as follows:

$f_{\theta}\left(X^{(l)}\right)=\left\langle W, X^{(l)}\right\rangle+b$

$p_{\theta}(a \mid X)=\frac{e^{f_{\theta}\left(X^{(a)}\right)}}{\sum_{l=1}^{K} e^{f_{\theta}\left(X^{(l)}\right)}}$

where $p_{\theta}(a \mid X)$ is the probability of the related ERP response after each stimulus. To predict the target, we maximized the posterior probability $p(a \mid X)$ given $X$ with respect to $\alpha$, then the target is the maximum output of the model $f_{\theta}\left(X^{(l)}\right)$ as follows:

$\hat{a}=\operatorname{arc} \max _{a} \log p_{\theta}(a \mid X)=\operatorname{arc} \max _{a} f_{\theta}\left(X^{(a)}\right)$

In our study, the collected EEG signals for all trials were band-pass filtered between 0.5 and $20 \mathrm{~Hz}$. The data epochs corresponding to each stimulus were extracted from 0 to $600 \mathrm{~ms}$ after stimulus onset. All epochs were re-referenced to the mean amplitude in the baseline, 100 to $0 \mathrm{~ms}$ before stimulus onset. We down-sampled the data epoch by a rate of 6 in each channel and the down-sampled epoch contained 25 data points. A feature vector $X^{(l)}$, with a length of 750 (30 channel $\times 25$ data points), was constructed by concatenating the data points of each channel. The vector $w$ was re-shaped from the coefficient matrix $W$ using a similar process. Therefore, the detection model becomes:

$f\left(X^{(l)}\right)=w^{T} X^{(l)}+b$

$\hat{a}=\operatorname{arc} \max _{a} f\left(X^{(a)}\right)$

A linear Support Vector Machine classifier was used to solve this model with training data. For the online test, the model (Eq. 4) was applied to predict the target stimulus that corresponded to a high score. Finally, the ratio of the number of trials with correct responses to the total number of trials in online blocks was calculated as the online accuracy of the BCI assessment

\section{Statistical Analysis}

To obtain the significance level of the accuracy, the $\chi^{2}$ statistical test was used. Specifically, the $\chi^{2}$ statistic was calculated as follows [19]:

$\chi^{2}=\sum_{i=1}^{k} \frac{\left(f_{o i}-f_{e i}\right)^{2}}{f_{e i}}$

where $f_{o i}$ and $f_{e i}$ are the observed and expected frequencies of the $i$ th class $(i=1,2, \ldots, k)$. In this study, a target was determined from the four flashing buttons. Therefore, the chance level that the target was selected was 0.25 , whereas the chance level for selecting a non-target was 0.75 . Considering that 20 trials of the online BCI test were conducted for each participant, the expected $f_{e 1}$ was 5 and the expected $f_{e 2}$ was 15 . Furthermore, $f_{o 1}$ and $f_{o 2}$ were the numbers of times that the target $(i=1)$ or a non-target $(i=2)$ was determined in the online BCI test. Using a significance level of $P=0.05$, we obtained a value of 3.84 for the $\chi^{2}$ test (degrees of freedom: 1), which corresponded to 9 hits in 20 trials or an accuracy of $45 \%$. Therefore, a participant was considered visual fixation responsive in the BCI assessment if he/she obtained a significant BCI accuracy.

\section{Results}

\section{Results for Healthy Participants}

The averaged online accuracy of BCI assessment in the healthy group was $84 \% \pm 3.4 \%$. Furthermore, each healthy participant achieved a significant accuracy (significance level: $45 \%, P<0.001, \chi^{2}$-test), as detailed in Table 2 . The effectiveness of our BCI system for visual fixation detection was thus demonstrated.

Offline ERP analysis was performed to further demonstrate the physiological plausibility of the proposed BCI system. We calculated the temporal ERP waveforms by averaging the 40 trials of EEG epochs for each participant. The group-averaged ERP waveforms were also obtained by averaging them across all 5 participants. The groupaveraged ERP waveforms of healthy participants from $\mathrm{FCz}, \mathrm{Cz}, \mathrm{Pz}, \mathrm{O} 1, \mathrm{Oz}$, and $\mathrm{O} 2$ are shown in Fig. 2. We first found a positive component between 300 and $400 \mathrm{~ms}$ in $\mathrm{FCz}, \mathrm{Cz}$, and $\mathrm{Pz}$, and a broad positive component between $350 \mathrm{~ms}$ and $600 \mathrm{~ms}$ in $\mathrm{O} 1, \mathrm{Oz}$, and $\mathrm{O} 2$, which was associated with P300. There was a negative component at $200 \mathrm{~ms}$ defined as the motion-specific visual evoked potential N200 in the six selected channels. The point-wise running $t$-test was also used to compare the difference between the responses elicited by target and non-target, and multiple comparison correction with a cluster size of 7 was used [20-22]. There were remarkable differences between the responses elicited by the target and non-target stimuli in the period $300-400 \mathrm{~ms}$ in $\mathrm{FCz}, \mathrm{Cz}$, and $\mathrm{Pz}$, and in the periods $200-300 \mathrm{~ms}$ and $350-500 \mathrm{~ms}$ in $\mathrm{O} 1, \mathrm{Oz}$, and $\mathrm{O} 2$ (Fig. 2A). The N200 and P300 components were clearly elicited by the target stimuli. The N200 occurred slightly more than $200 \mathrm{~ms}$ after stimulus onset and was significant in $\mathrm{O} 1, \mathrm{Oz}$, and $\mathrm{O} 2$. The $\mathrm{P} 300$ with a latency between 300 and $500 \mathrm{~ms}$ was significant in the six channels and was broad in $\mathrm{O} 1, \mathrm{Oz}$, and $\mathrm{O} 2$. Furthermore, no ERP component was elicited by the non-target stimuli, and this result 
Table 2 Online results of healthy participants.

\begin{tabular}{lllc}
\hline Healthy participants & Number of trials & BCI online accuracy $(\%)$ & $P$ value $\left(\chi^{2}\right.$-test $)$ \\
\hline HC1 & 20 & 80 & 0.0146 \\
HC2 & 20 & 85 & 0.0035 \\
HC3 & 20 & 85 & 0.0035 \\
HC4 & 20 & 90 & $<0.001$ \\
HC5 & 20 & 80 & 0.0146 \\
Mean \pm SD & 20 & $84 \pm 3.4$ & $<0.001$ \\
\hline
\end{tabular}

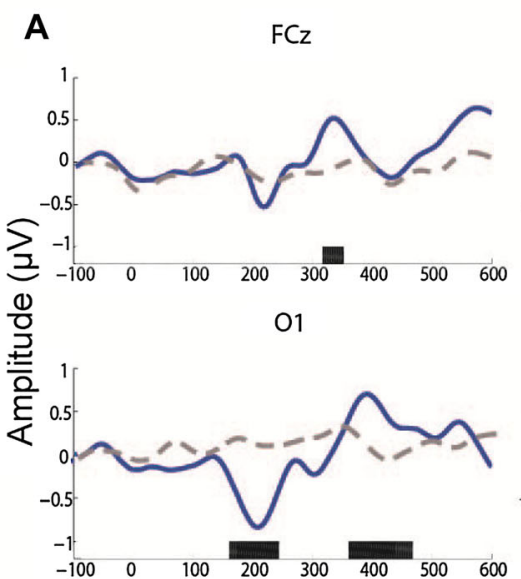

B
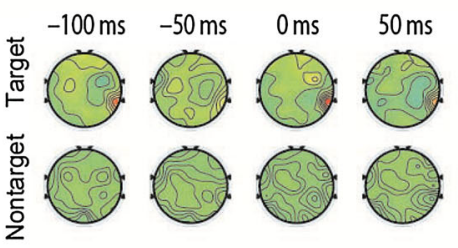

$\mathrm{Cz}$

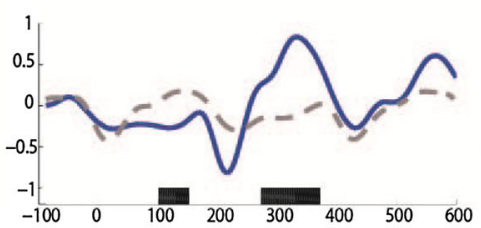

$\mathrm{Oz}$

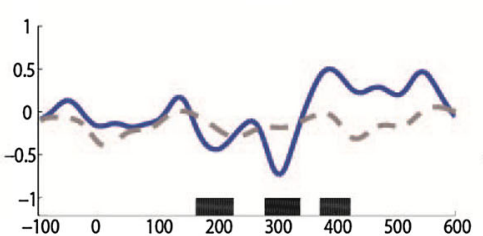

$\mathrm{Pz}$

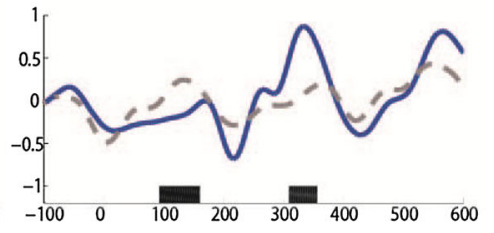

$\mathrm{O} 2$

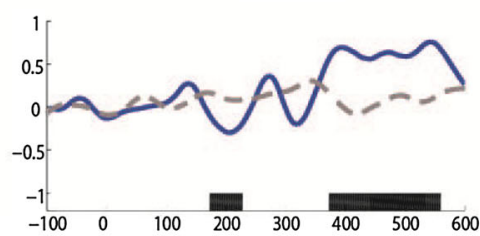

Time (ms)
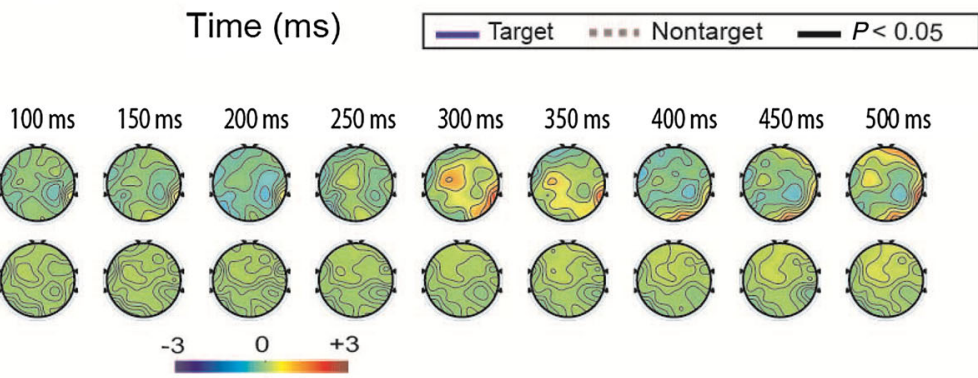

Fig. 2 Averaged ERP waveforms and scalp topography of five healthy participants. A Waveforms from $100 \mathrm{~ms}$ before the stimulus onset to $600 \mathrm{~ms}$ after stimulus onset in the six channels $(\mathrm{FCz}, \mathrm{Cz}, \mathrm{Pz}$, $\mathrm{O} 1, \mathrm{Oz}$, and $\mathrm{O} 2$ ). Black bars, time intervals with significant

differences of the waveforms evoked by target and non-target (twosample $t$-test, $P<0.05$ ). B Scalp topography averaged from the 5 healthy participants.

coincided with the physiological principles in reported references [23-27]. The averaged scalp topography of the 5 healthy participants is also shown in Fig. 2B. We found that the negative component $\mathrm{N} 200$ was distributed in the temporo-occipital and associated parietal areas between 200 and $250 \mathrm{~ms}$. The P300 was distributed in the central regions between 300 and $350 \mathrm{~ms}$. The online accuracy rates, the apparent ERP waveforms, and reasonable distribution in scalp topography of the healthy participants validated the effectiveness of the BCI paradigm.

\section{Results from Patients}

The 15 DOC patients participated in the BCI experiment over 7 months; their online results are shown in Table 3. In the BCI assessment, an online accuracy $>45 \%$ was considered to show visual fixation. In the CRS-R assessment, a visual subscale score (the second sub-score in the CRS-R scores) of 2 means that visual fixation behavior was detected by the clinician.

First, among the 15 patients, patients 12,13 , and 15 achieved significant accuracy and were considered to be 
Table 3 BCI online and CRS$\mathrm{R}$ results for DOC patients.

\begin{tabular}{llllll}
\hline Patient & Trials & Hits & BCI online accuracy $(\%)$ & $P$ value & CRS-R score \\
\hline Patient 1 & 20 & 4 & 20 & 0.61 & $4(1-0-1-0-0-2)$ \\
Patient 2 & 20 & 5 & 25 & 1 & $5(1-0-2-0-0-2)$ \\
Patient 3 & 20 & 6 & 30 & 1 & $5(1-1-0-1-0-2)$ \\
Patient 4 & 20 & 4 & 20 & 0.61 & $5(1-0-1-1-0-2)$ \\
Patient 5 & 20 & 5 & 25 & 1 & $6(1-1-1-1-0-2)$ \\
Patient 6 & 20 & 8 & 40 & 0.24 & $7(1-1-2-1-0-2)$ \\
Patient 7 & 20 & 4 & 20 & 0.61 & $7(1-0-2-2-0-2)$ \\
Patient 8 & 20 & 11 & $\mathbf{5 5}$ & 0.0019 & $7(1-1-2-1-0-2)$ \\
Patient 9 & 20 & 8 & 40 & 0.24 & $9(1-1-4-1-0-2)$ \\
Patient 10 & 20 & 7 & 35 & 0.61 & $9(1-1-4-1-0-2)$ \\
Patient 11 & 20 & 5 & 25 & 1 & $10(1-3-3-1-0-2)$ \\
Patient 12 & 20 & 9 & $\mathbf{4 5}$ & 0.04 & $11(1-3-5-0-0-2)$ \\
Patient 13 & 20 & 9 & $\mathbf{4 5}$ & 0.04 & $15(2-5-5-1-0-2)$ \\
Patient 14 & 20 & 7 & 35 & 0.61 & $20(4-5-6-1-2-2)$ \\
Patient 15 & 20 & 13 & $\mathbf{6 5}$ & $<0.001$ & locked-in syndrome \\
\hline
\end{tabular}

Accuracies $>45 \%\left(P=0.05, \chi^{2}\right.$ test $)$ are marked in bold. responsive to visual fixation in the $\mathrm{BCI}$ assessment. The visual subscale scores were 3 for patient 12 and 5 for patient 13, indicating that higher visual behavior was detected by CRS-R. Patient 15 (locked-in syndrome) had recovered consciousness and showed consistent commandfollowing. Thus, the three patients were classified into the responsive group by both BCI and CRS-R. Second, 9 of the 15 patients (patients $1,2,3,4,5,6,7,9$, and 10), who did not show visual fixation in either the BCI or the CRS-R assessment, were classified into the non-responsive group. In this group, patients 1, 2, 4, and 7 failed to show visual fixation. Clinical assessment showed that these four patients failed to show eye-blinks in response to threat and their visual subscale scores were 0 (Table 3 ), indicating impaired brainstem reflexes. Patients 3, 5, 6, 9, and 10 had a visual subscale score of 1 , which implied that visual fixation was not detected by the CRS-R behavioral scale. Third, patients 11 and 14 did not achieve significant accuracy in the BCI assessment but showed higher visual behavior (patient 11) or cognitive behavior (patient 14) in CRS-R assessment. Patient 11 showed visual pursuit behavior, a higher behavior item than visual fixation in CRS-R. Patient 14 showed higher cognitive functions and was considered conscious in the clinical assessment. However, their BCI online accuracy was close to the chance level of $25 \%$. So patients 11 and 14 were classified into the non-responsive group by BCI assessment (BCInon-responsive group). Finally and more importantly, patient 8 showed no visual fixation in the CRS-R measurement before the BCI measurement, but achieved significant online accuracy in the BCI assessment (Table 3) and was classified into the responsive group by BCI (BCIresponsive group).
In patients 12,13 , and 15 in the responsive group, the averaged ERP waveforms and scalp topography were also obtained by averaging all 40 trials for each patient (Fig. 3). In patient 12 , a broad positive component related to P300 was exhibited in all six channels, and there were significant differences between the waveforms elicited by the target and non-target stimuli in the period from $200 \mathrm{~ms}$ to $300 \mathrm{~ms}$ in $\mathrm{FCz}, \mathrm{Cz}$, and $\mathrm{O} 1$ and in the period from $100 \mathrm{~ms}$ to 400 $\mathrm{ms}$ in $\mathrm{Pz}, \mathrm{Oz}$, and $\mathrm{O} 2$ (Fig. 3A). In addition, positive components were found over the whole scalp between 350 $\mathrm{ms}$ and $500 \mathrm{~ms}$ (Fig. 3A, lower panels). In patient 13, there were negative components in $\mathrm{FCz}$ and $\mathrm{Cz}$, but a tiny positive peak occurred at $300 \mathrm{~ms}$ in $\mathrm{Pz}, \mathrm{O} 1, \mathrm{Oz}$, and $\mathrm{O} 2$. Remarkable differences between the responses elicited by the target and non-target stimuli were not observed throughout the period from $100 \mathrm{~ms}$ to $600 \mathrm{~ms}$, except at $0 \mathrm{~ms}$ in Oz (Fig. 3B). The scalp map showed a negative component distributed in the lateral temporal and central areas. After $300 \mathrm{~ms}$, a positive component was generated from the central and associated occipital sites (Fig. 3B, lower panels). In the locked-in syndrome patient 15, there was a significant positive peak in the range $200-300 \mathrm{~ms}$ in $\mathrm{Cz}$ and $\mathrm{O} 1$, and a broad positive component between 200 $\mathrm{ms}$ and $600 \mathrm{~ms}$ in Pz, especially in the periods $200-400 \mathrm{~ms}$ and 500-600 ms (Fig. 3C). The ERP component associated with P300 emerged between 300 and $500 \mathrm{~ms}$ and was distributed in the centro-parietal regions (Fig. 3C, lower panels). These findings are consistent with the neurophysiological results reported in previous publications [27-30].

However, the patients in the non-responsive group and BCI-nonresponsive group did not have any visual fixation response in the BCI assessment. Moreover, there was no corresponding ERP component in these patients' individual 
A
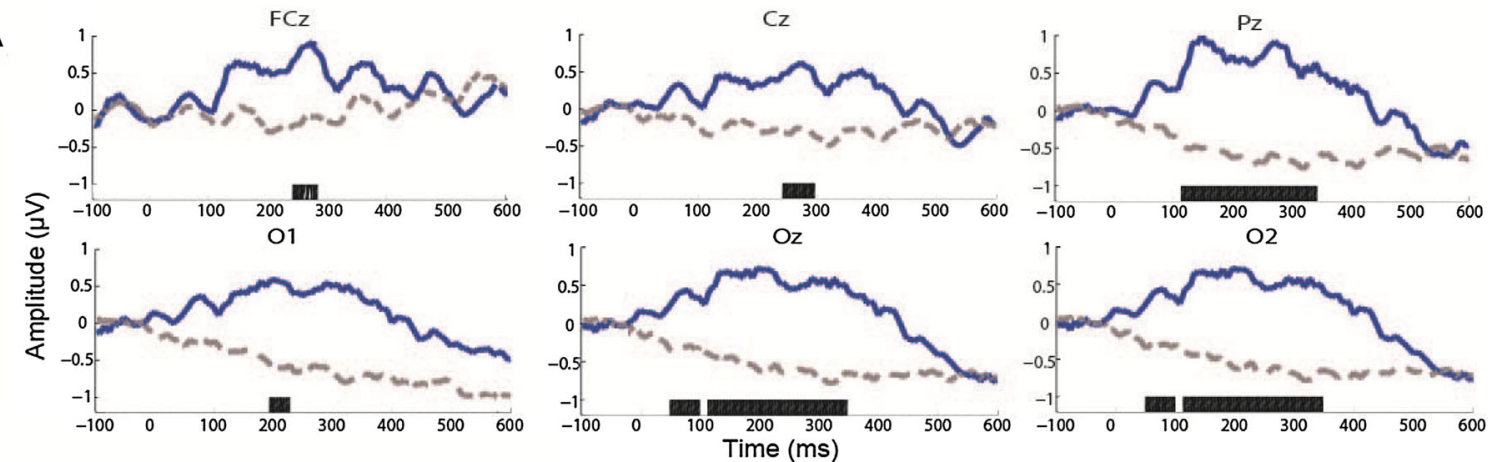
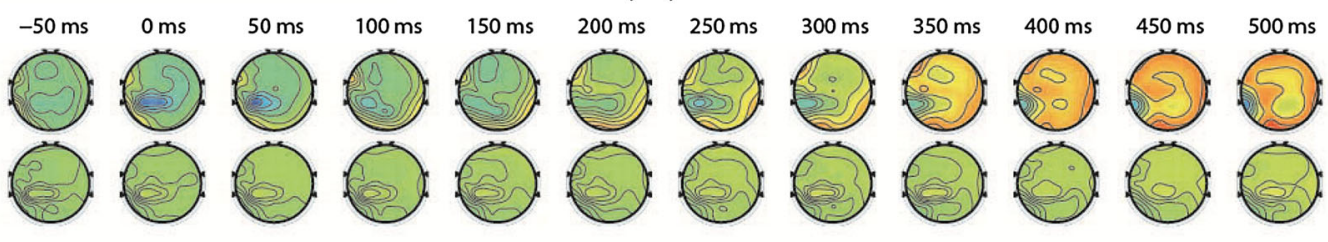

B
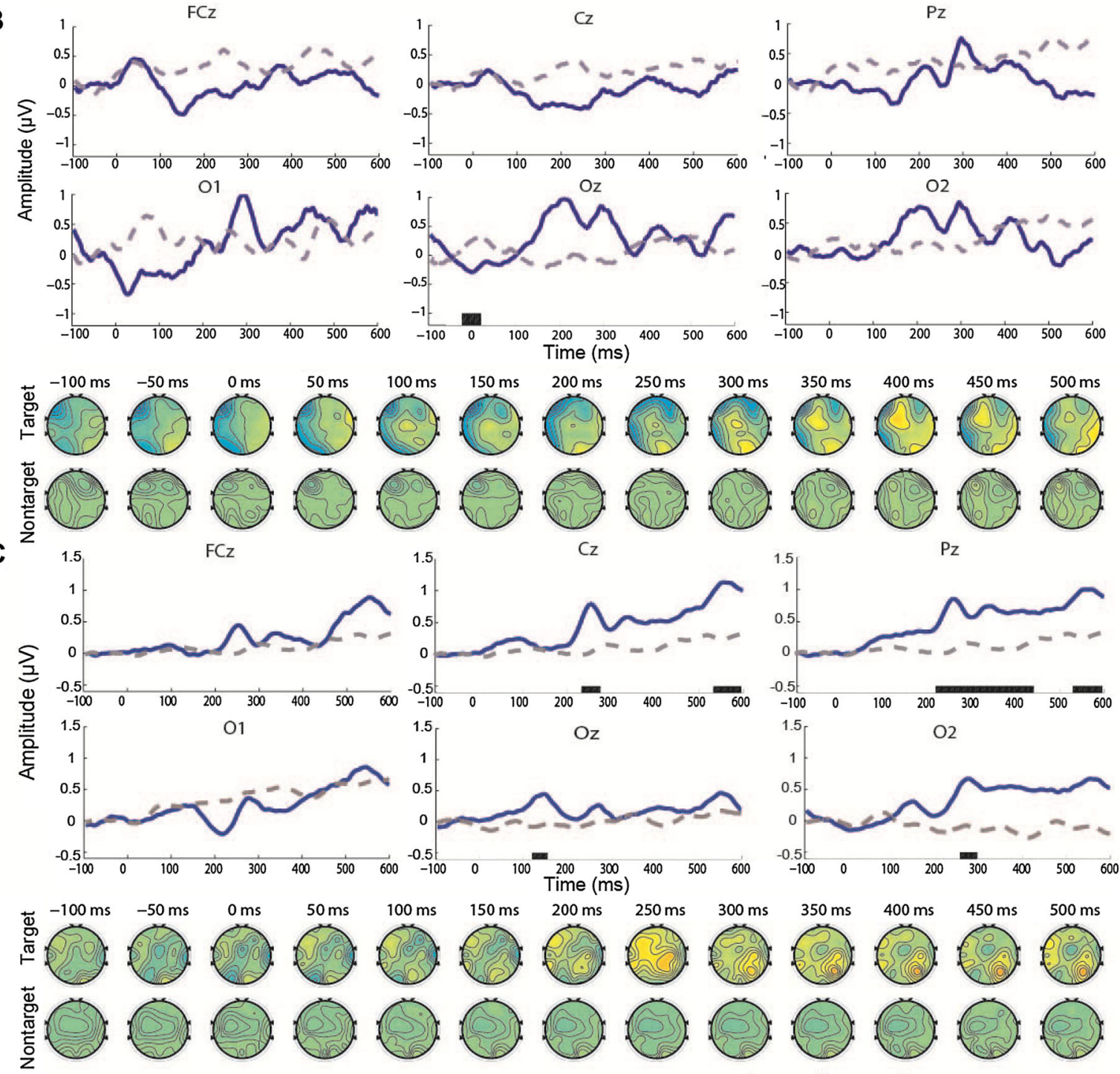

- Target $\quad \cdots \cdots$ Nontarget $\quad-P<0.05$ 
4 Fig. 3 A-C ERP waveforms and scalp topography in patients 12 (A), 13 (B), and 15 (C). The waveforms were obtained from $100 \mathrm{~ms}$ before the stimulus onset to $600 \mathrm{~ms}$ after the stimulus onset in six channels ( $\mathrm{FCz}, \mathrm{Cz}, \mathrm{Pz}, \mathrm{O} 1, \mathrm{Oz}$, and $\mathrm{O} 2$ ). The blue solid and gray dashed lines denote the waveforms evoked by the target and non-target stimuli, respectively.

ERP waveforms. The clinical results from patients in the non-responsive group were consistent with the $\mathrm{BCI}$ results. The patients in the BCI-nonresponsive group, including patients 11 and 14, showed higher visual function but no visual fixation by $\mathrm{BCI}$ assessment.

Interestingly, patient 8 showed no visual fixation behavior in the CRS-R measurement but achieved significant online accuracy in the BCI assessment (Table 3). He showed a significant positive peak in the range $200-400 \mathrm{~ms}$ in $\mathrm{FCz}, \mathrm{Cz}$, and $\mathrm{Pz}$, distributed in the frontal-central, central, and related parietal regions, and a negative component after $350 \mathrm{~ms}$ in the occipital area (Fig. 4).

\section{Discussion}

In this study, a visual BCI system was developed to assist visual fixation assessment in patients with DOCs. Specifically, this BCI system was designed to simulate the behavioral evaluation of visual fixation in the CRS-R, in which a button (similar to the moving object in the behavioral assessment) was presented in the center of the GUI and moved to a new target position. The moving button aided patients to re-fixate on the target position. The patients were instructed to follow the moving button and re-fixate on the new position while their EEG signals were collected. Based on the EEG data, the BCI algorithm determined whether the patient re-fixated on the target. The efficacy of the proposed system was first demonstrated by an experiment involving five healthy participants. Then, 15 patients with DOCs participated in the assessments based on the BCI system and the CRS-R. Among the 15 patients, three who exhibited higher visual function in the CRS-R assessment were also found to be visual fixation responsive in the BCI assessment. The corresponding ERP components were evident in their ERP waveforms. However, 11 out of 15 patients did not exhibit visual fixation in the BCI assessment. Specifically, 2 of 11 patients exhibited visual pursuit and command following clinical assessment. Nine of the eleven patients did not show visual fixation behavior in CRS-R behavioral assessment. More importantly, one patient did not show visual fixation behavior in CRS-R but exhibited visual fixation in BCI measurement.

Motor disability poses a practical challenge for clinicians working with DOC patients in terms of diagnosis, care, and rehabilitation [31-33]. In clinical assessment, the behavioral scale, which remains the traditional way to evaluate consciousness in these patients, is highly dependent on motor abilities. The BCI may provide a method to
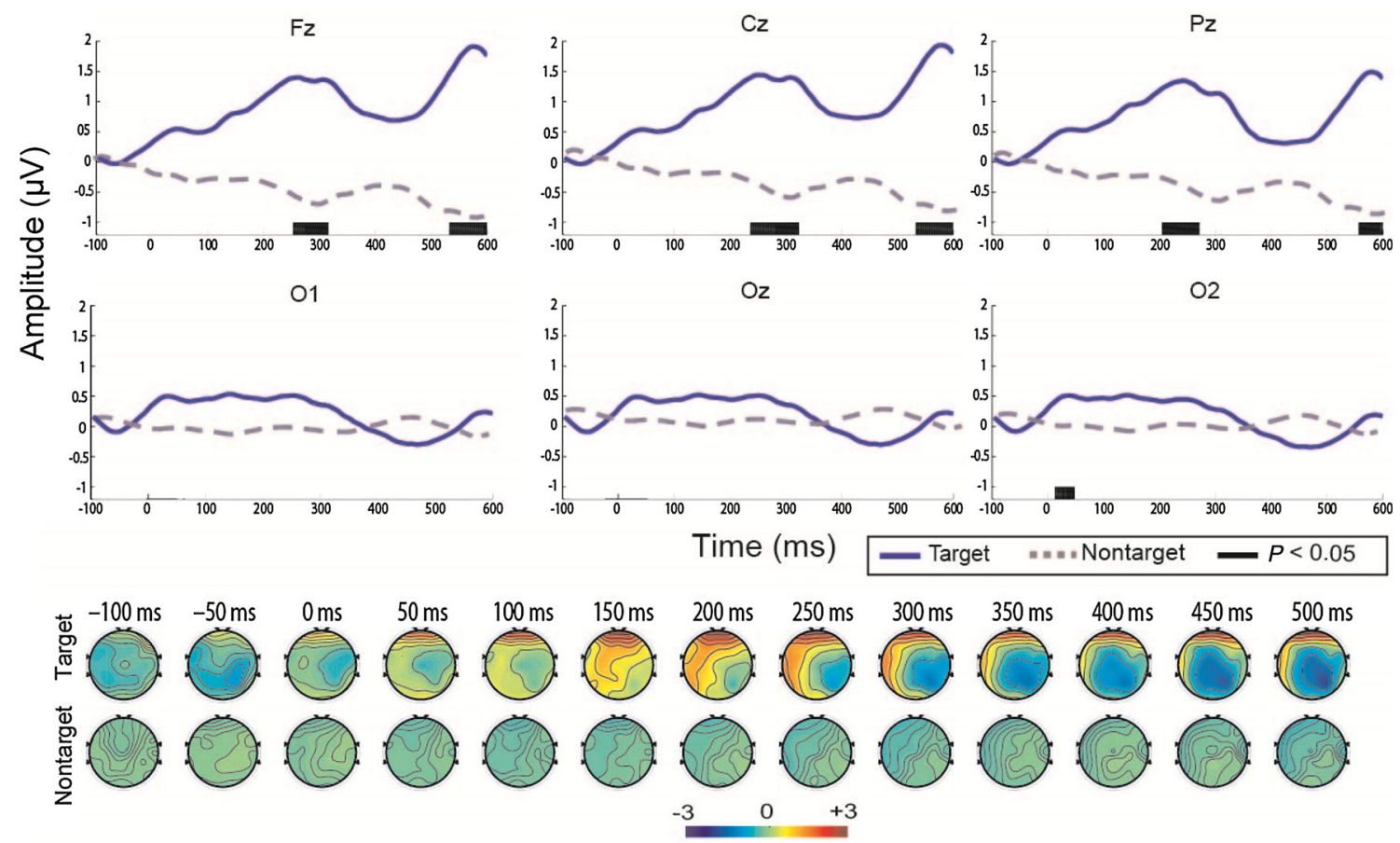

Fig. 4 ERP waveforms and scalp topography in patient 8. The waveforms were obtained from $100 \mathrm{~ms}$ before the stimulus onset to $600 \mathrm{~ms}$ after the stimulus onset in the six channels $(\mathrm{FCz}, \mathrm{Cz}, \mathrm{Pz}, \mathrm{O} 1$,
$\mathrm{Oz}$, and $\mathrm{O} 2$ ). The blue solid and gray dashed lines denote the waveforms evoked by the target and non-target stimuli, respectively. 
directly detect the endogenous brain response of DOC patients to external stimuli, independent of behavioral expression. Recently, the "command-following" or "communication" BCI paradigm has helped to reduce the possibility of misdiagnosis of those DOC patients who have severely impaired motor abilities [31, 34, 35]. However, this type of protocol still requires high-level cognitive abilities, such as language understanding and task switching, compared to behavioral assessment. Therefore, poor performance and a high rate of false-negatives (patients showing command-following at the beside but not detected with BCI; 22\%-94\%) [36] are well-known limitations of BCIs for detecting command-following and communication in DOC patients. This issue highlights the current need to develop more reliable tools for the diagnosis of patients with DOCs. This study was designed to explore the feasibility of using visual BCI systems to complement the visual fixation behavioral assessments. The proposed BCI system was designed to simulate the behavioral visual fixation evaluation in CRS-R, the four buttons in different locations flashed and elicited the time-locked features of ERP components. The combination of CRS-R and BCI assessment may help to provide more precise, objective, and sensitive diagnoses. In this manner, the BCI's poor performance can be improved because our system did not require that the patient have much more capacity than required by the current $\mathrm{BCI}$ for detecting commandfollowing and communication.

For the proposed novel BCI paradigm, the motion of stimuli embedded into onscreen virtual buttons and the rare but task-relevant flashes have been applied. Based on the paradigm, the corresponding ERP components including the motion-onset visual evoked potential N200 and P300 components were elicited by the stimuli as reported previously [23, 37]. Compared to the traditional P300based BCI system, the increased ERP component N200 may help to improve the classification accuracy of the BCI. From the results of healthy subjects, the negative component (between 200 and $250 \mathrm{~ms}$ ) of the specific N200 was found in the temporo-occipital and associated parietal cortical areas, including $\mathrm{O} 1, \mathrm{Oz}$, and Pz. A clear P300 component emerged in the central and parietal regions, including $\mathrm{Cz}$ and $\mathrm{Pz}$. Furthermore, a positive component after $500 \mathrm{~ms}$ that resembled the late positive component was exhibited in $\mathrm{O} 1$ and $\mathrm{O} 2$. There was no negative component in the ERP waveforms of the patients in the responsive group, but a broad $\mathrm{P} 300$ component was evident in each patient's waveform. The differences in ERP waveforms between the healthy participants and DOC patients might be due to the severe brain injuries and limited cognitive levels of these patients.

In summary, among the 15 patients, two (patients 11 and 14) showed visual pursuit (higher visual function) or cognitive behavior in CRS-R assessment, but visual fixation was not detected by the BCI system. Actually, this phenomenon has been discussed in previous studies of visual fixation assessment [6]. According to the guidelines for assessing visual fixation in the CRS-R (e.g., use an object), many DOC patients fail to show visual fixating behavior while higher cognitive functions are present [6]. Our BCI system simulated the standard protocol in CRS-R, using a brightly-colored ball as the visual stimulus. Therefore, the phenomenon also occurred in our BCI system for visual fixation assessment; changing the visual stimulus described in the standard protocol (i.e., a colored ball or a bright light) may improve the detection of visual fixation such that the accuracy of BCI assessment is also improved. More importantly, one patient who did not show visual fixation behavior was judged to be visual fixation responsive by the BCI assessment. This result indicated that the BCI method may assist the clinical assessment of visual fixation in patients with DOCs and alleviate the risk of misdiagnosis.

The proposed BCI system aimed to complement the classical behavioral assessment for DOC patients. Therefore, the score on the visual fixation item should be revised according to the results of CRS-R and BCI. (1) If the DOC patient obtains consistent results (positive or negative) from both CRS-R and BCI, the item score of visual fixation remains unchanged. Specifically, if a reliable and consistent visual fixation behavior is detected by CRS-R and the accuracy of BCI is higher than the significance level, the score of visual fixation is 2 . Conversely, if the results from both assessments are negative, the patient lacks visual fixation behavior or both methods missed it. The score is $<2$ and may depend on assessment of the lower item (e.g., visual startle) than the visual fixation. (2) If a repeatable visual fixation behavior is detected by CRS-R but the result of the BCI is negative (missed), then the CRS-R result is preferred because a proportion of people are $\mathrm{BCI}$ illiteracy, which is that BCI control does not work for a few of users. (3) If visual fixation behavior is not observed in the patient by CRS-R but a significant accuracy rate in the BCI test is achieved, then the BCI result has priority and the score of visual fixation item is revised to 2 .

This pilot study proposed a novel BCI system that provides assessment of visual fixation in DOC patients. In contrast to previous studies of BCI detection of consciousness, which still requires high-level cognitive abilities, we designed a BCI system simulating the visual fixation behavioral assessment. Combining the CRS-R- and BCIbased assessments may provide a degree of correction for the behavioral evaluation. In future, some improvements can be made. First, the averaged online accuracy of healthy subjects was $84 \% \pm 3.4 \%$, while the highest online accuracy of responsive patients judged by the BCI system 
only achieved $65 \%$. Four responsive patients in BCI assessment had specific ERP patterns and showed different temporal properties. Furthermore, we used no special method for reducing artifacts in this study, as in many studies. Future investigators may consider how to design a feature-selection algorithm (e.g., channel selection) to be adapted to these patients for improving the detection performance of our BCI system and the preprocessing method can be elaborated to reduce artifacts in the EEG signals. Second, increasing the number of participants is underway for improving the feasibility of our system in clinical applications. Third, developing an integrated BCI system based on several assessment items of the CRS-R from our previous work is our goal in the future.

Acknowledgements This work was supported by the National Key Research and Development Program of China (2017YFB1002505), the National Natural Science Foundation of China (61633010, 91420302, and 61503143), and the Natural Science Foundation of Guangdong Province, China (2014A030312005 and 2014A030310244), and the Pearl River S\&T Nova Program of Guangzhou Municipality, China (201710010038).

\section{Compliance with Ethical Standards}

Conflict of interest All authors claim that there are no conflicts of interest.

Open Access This article is distributed under the terms of the Creative Commons Attribution 4.0 International License (http:// creativecommons.org/licenses/by/4.0/), which permits unrestricted use, distribution, and reproduction in any medium, provided you give appropriate credit to the original author(s) and the source, provide a link to the Creative Commons license, and indicate if changes were made.

\section{References}

1. Giacino JT, Kalmar K, Whyte J. The JFK Coma Recovery ScaleRevised: measurement characteristics and diagnostic utility. Arch Phys Med Rehabil 2004, 85: 2020-2029.

2. La Porta F, Caselli S, Ianes AB, Cameli O, Lino M, Piperno R, et al. Can we scientifically and reliably measure the level of consciousness in vegetative and minimally conscious States? Rasch analysis of the coma recovery scale-revised. Arch Phys Med Rehabil 2013, 94: 527-535.

3. Schnakers C, Vanhaudenhuyse A, Giacino J, Ventura M, Boly M, Majerus S, et al. Diagnostic accuracy of the vegetative and minimally conscious state: clinical consensus versus standardized neurobehavioral assessment. BMC Neurol 2009, 9: 35.

4. Giacino JT, Schnakers C, Rodriguez-Moreno D, Kalmar K, Schiff N, Hirsch J. Behavioral assessment in patients with disorders of consciousness: gold standard or fool's gold? Prog Brain Res 2009, 177: 33-48.

5. Bruno MA, Vanhaudenhuyse A, Schnakers C, Boly M, Gosseries $\mathrm{O}$, Demertzi A, et al. Visual fixation in the vegetative state: an observational case series PET study. BMC Neurol 2010, 10: 1-6.

6. Di $\mathrm{H}$, Nie $\mathrm{Y}, \mathrm{Hu} \mathrm{X}$, Tong $\mathrm{Y}$, Heine $\mathrm{L}$, Wannez $\mathrm{S}$, et al. Assessment of visual fixation in vegetative and minimally conscious states. BMC Neurol 2014, 14: 1-6.
7. Naro A, Leo A, Buda A, Manuli A, Bramanti A, Bramanti P, et al. Do you see me? The role of visual fixation in chronic disorders of consciousness differential diagnosis. Brain Res 2016, 1653: 59-66.

8. Vanhaudenhuyse A, Schnakers C, Brédart S, Laureys S. Assessment of visual pursuit in post-comatose states: use a mirror. J Neurol Neurosurg Psychiatry 2008, 79: 177-177.

9. Luaut EJ, Morlet D, Mattout J. BCI in patients with disorders of consciousness: clinical perspectives. Ann Phys Rehab Med 2015, 58: 29-34.

10. Majerus S, Gill-Thwaites H, Andrews K, Laureys S. Behavioral evaluation of consciousness in severe brain damage. Prog Brain Res 2005, 150: 1842-1857.

11. Childs NL, Mercer WN, Childs HW. Accuracy of diagnosis of persistent vegetative state. Neurology 1993, 43: 1465-1467.

12. Cortese MD, Riganello F, Arcuri F, Pugliese ME, Lucca LF, Dolce $\mathrm{G}$, et al. Coma recovery scale-r: variability in the disorder of consciousness. BMC Neurology 2015, 15: 1-7.

13. Schnakers C, Vanhaudenhuyse A, Giacino J, Ventura M, Boly M, Majerus $\mathrm{S}$, et al. Diagnostic accuracy of the vegetative and minimally conscious state: Clinical consensus versus standardized neurobehavioral assessment. BMC Neurol 2009, 9: 1-5.

14. Andrews K, Murphy L, Munday R, Littlewood C. Misdiagnosis of the vegetative state: retrospective study in a rehabilitation unit. BMJ 1996, 313: 13-16.

15. Xiao J, Xie Q, He Y, Yu T, Lu S, Huang N, et al. An auditory BCI system for assisting CRS-R behavioral assessment in patients with disorders of consciousness. Sci Rep 2016, 6: 32917.

16. Wang F, He Y, Qu J, Xie Q, Lin Q, Ni X, et al. Enhancing clinical communication assessments Using an audiovisual BCI for patients with disorders of consciousness. J Neural Eng 2017, 14: 046024. .

17. $\mathrm{Yu} \mathrm{T}, \mathrm{Yu} \mathrm{Z}, \mathrm{Gu} \mathrm{Z}, \mathrm{Li} \mathrm{Y}$. Grouped automatic relevance determination and its application in channel selection for P300 BCIs. IEEE Trans Neural Syst Rehabil Eng 2015, 23: 1068-1079.

18. Polich J, Criado JR. Neuropsychology and neuropharmacology of P3a and P3b. Int J Psychophysiol 2006, 60: 172-185.

19. Kübler A, Birbaumer N. Brain computer interfaces and communication in paralysis: extinction of goal directed thinking in completely paralysed patients? Clin Neurophysiol 2008, 119: $2658-2666$.

20. Senkowski D, Saint-Amour D, Höfle M, Foxe JJ. Multisensory interactions in early evoked brain activity follow the principle of inverse effectiveness. Neuroimage 2011, 56: 2200-2208.

21. Senkowski $D^{1}$, Saint-Amour D, Kelly SP, Foxe JJ. Multisensory processing of naturalistic objects in motion: a high-density electrical mapping and source estimation study. Neuroimage 2007, 36: 877-888.

22. Guthrie D, Buchwald JS. Significance testing of difference potentials. Psychophysiology 1991, 28: 240-244.

23. Guo F, Hong B, Gao X, Gao S. A brain-computer interface using motion-onset visual evoked potential. J Neural Eng 2008, 5: $477-485$.

24. Näätänen R, Gaillard AW, Mäntysalo S. Early selective-attention effect on evoked potential reinterpreted. Acta Psychol (Amst) 1978, 42: 313-329.

25. Marzi T, Viggiano MP. Interplay between familiarity and orientation in face processing: An ERP study. Int J Psychophysiol 2007, 65: 182.

26. Vidal JJ. Real-time detection of brain events in EEG. Proceedings of the IEEE 1977, 65: 633-641.

27. Monti MM, Vanhaudenhuyse A, Coleman MR, Boly M, Pickard JD, Tshibanda L, et al. Willful modulation of brain activity in disorders of consciousness. N Engl J Med 2010, 362: 579-589.

28. Duncan CC, Barry RJ, Connolly JF, Fischer C, Michie PT, Näätänen $\mathrm{R}$, et al. Event-related potentials in clinical research: 
guidelines for eliciting, recording, and quantifying mismatch negativity, P300, and N400. Clin Neurophysiol 2009, 120: 1883-1908.

29. Guérit JM, Verougstraete D, de Tourtchaninoff M, Debatisse D, Witdoeckt C. ERPs obtained with the auditory oddball paradigm in coma and altered states of consciousness: clinical relationships, prognostic value, and origin of components. Clin Neurophysiol 1999, 110: 1260-1269.

30. Polich J. Updating P300: an integrative theory of P3a and P3b. Clin Neurophysiol 2007, 118: 2128-2148.

31. Schnakers C, Perrin F, Schabus M, Hustinx R, Majerus S, Moonen G, et al. Detecting consciousness in a total locked-in syndrome: an active event-related paradigm. Neurocase 2009, 15: 271-277.

32. Martin MM, Vanhaudenhuyse A, Coleman MR, Boly M, Pickard JD, Tshibanda L, et al. Willful modulation of brain activity in disorders of consciousness. N Engl J Med 2010, 362: 1937-1938.
33. Owen AM, Coleman MR. Detecting awareness in the vegetative state. Ann N Y Acad Sci 2006, 315: 130-138.

34. Pan J, Xie Q, He Y, Wang F, Di H, Laureys S, et al. Detecting awareness in patients with disorders of consciousness using a hybrid brain-computer interface. J Neural Eng 2014, 11: 056007.

35. Lule D, Noirhomme Q, Kleih SC, Chatelle C, Halder S, Demertzi A, et al. Probing command following in patients with disorders of consciousness using a brain-computer interface. Clin Neurophysiol 2013, 124: 101-106.

36. Chatelle C, Lesenfants D, Guller Y, Laureys S, Noirhomme Q. Brain-Computer Interface for Assessing Consciousness in Severely Brain-Injured Patients. Springer Vienna, 2015: 133-148. 